\title{
A variant in the CD209 promoter is associated with severity of dengue disease
}

Anavaj Sakuntabhai ${ }^{1,2}$, Chairat Turbpaiboon ${ }^{1,3}$, Isabelle Casadémont ${ }^{1}$, Ampaiwan Chuansumrit ${ }^{4}$, Tassanee Lowhnoo ${ }^{1,5,6}$, Anna Kajaste-Rudnitski ${ }^{7}$, Sita Mint Kalayanarooj ${ }^{1,8}$, Kanchana Tangnararatchakit ${ }^{4}$, Nattaya Tangthawornchaikul ${ }^{9}$, Sirijit Vasanawathana ${ }^{10}$, Wathanee Chaiyaratana ${ }^{6}$, Pa-thai Yenchitsomanus ${ }^{8,9}$, Prapat Suriyaphol $^{8}$, Panisadee Avirutnan ${ }^{8}$, Kulkanya Chokephaibulkit ${ }^{11}$, Fumihiko Matsuda ${ }^{5}$, Sutee Yoksan $^{12}$, Yves Jacob $^{13}$, G Mark Lathrop ${ }^{5}$, Prida Malasit ${ }^{8,9}$, Philippe Desprès $^{7}$ \& Cécile Julier ${ }^{1}$

Dengue fever and dengue hemorrhagic fever are mosquitoborne viral diseases. Dendritic cell-specific ICAM-3 grabbing nonintegrin (DC-SIGN1, encoded by CD209), an attachment receptor of dengue virus, is essential for productive infection of dendritic cells ${ }^{1,2}$. Here, we report strong association between a promoter variant of CD209, DCSIGN1-336, and risk of dengue fever compared with dengue hemorrhagic fever or population controls. The $G$ allele of the variant DCSIGN1-336 was associated with strong protection against dengue fever in three independent cohorts from Thailand, with a carrier frequency of $4.7 \%$ in individuals with dengue fever compared with $22.4 \%$ in individuals with dengue hemorrhagic fever (odds ratio for risk of dengue hemorrhagic fever versus (2) dengue fever: $5.84, P=1.4 \times 10^{-7}$ ) and $19.5 \%$ in controls (odds ratio for protection: 4.90, $P=2 \times 10^{-6}$ ). This variant affects an Sp1-like binding site and transcriptional activity in vitro. These results indicate that $C D 209$ has a crucial role in dengue pathogenesis, which discriminates between severe dengue fever and dengue hemorrhagic fever. This may have consequences for therapeutic and preventive strategies.

We recruited independent cohorts from three hospitals in Thailand, for a total of 606 individuals with dengue disease and 696 healthy population controls from the same hospitals. Viral diagnosis was confirmed by serologic tests, and affected individuals were classified in five groups according to World Health Organization criteria: dengue fever (no evidence of plasma leakage) or dengue hemorrhagic fever (evidence of plasma leakage) with increasing severity from grade I to grade IV. The study was restricted to hospitalized schoolage children. For dengue fever, we restricted our study to the most

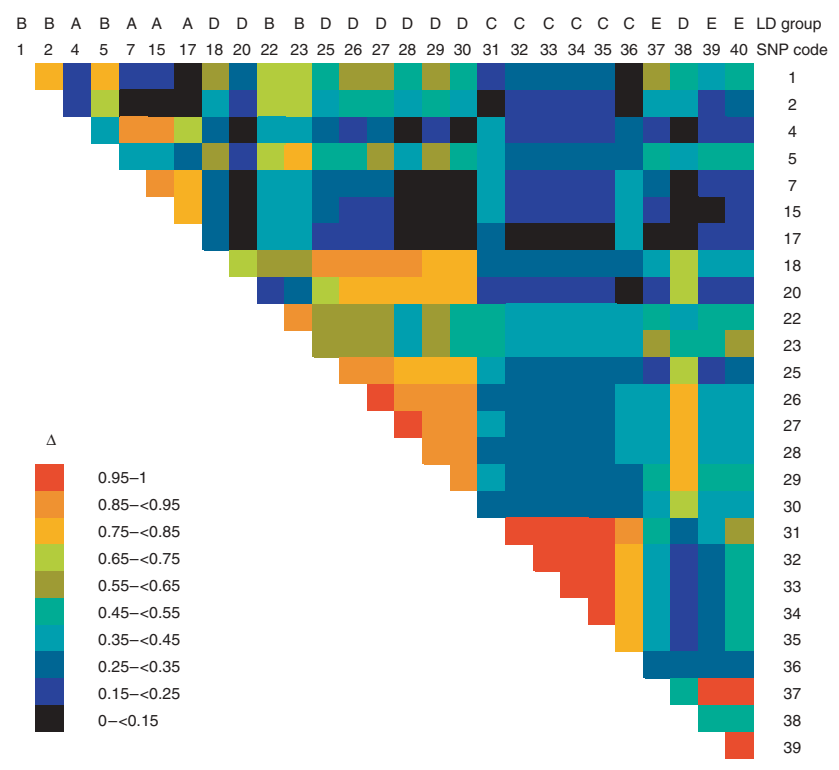

Figure 1 LD between CD209 polymorphisms in the Thai population. $\Delta$ values for SNPs with rare allele frequency $\geq 0.02$.

\footnotetext{
${ }^{1}$ Génétique des Maladies Infectieuses et Autoimmunes, Institut Pasteur, INSERM E102, 28 rue du docteur Roux, 75724 Paris Cedex 15, France. ${ }^{2}$ Department of Medicine, Faculty of Medicine, Ramathibodi Hospital, ${ }^{3}$ Department of Biochemistry, Faculty of Science and ${ }^{4}$ Department of Pediatrics, Faculty of Medicine, Ramathibodi Hospital, Mahidol University, Rama VI, Bangkok 10400, Thailand. ${ }^{5}$ Centre National de Génotypage, 2 rue Gaston Crémieux, CP 5721,91057 Evry Cedex, France. ${ }^{6}$ Research Center, Faculty of Medicine, Ramathibodi Hospital, Mahidol University, Rama VI, Bangkok 10400, Thailand. ${ }^{7}$ Interactions Moléculaires FlavivirusHôtes, Institut Pasteur, 25 rue du docteur Roux, 75724 Paris cedex 15, France. ${ }^{8}$ Medical Molecular Biology Unit, Faculty of Medicine, Siriraj Hospital, Mahidol University, Bangkok-noi, Bangkok 10700, Thailand. ${ }^{2}$ Medical Biotechnology Unit, National Center for Genetic Engineering and Biotechnology BIOTEC, National Science and Technology Development Agency NSTDA, Pathumthani 12120, Thailand. ${ }^{10}$ Department of Pediatrics, Khon Kaen Hospital, Ministry of Public Health, Khonkaen 40000, Thailand. ${ }^{11}$ Department of Pediatrics, Faculty of Medicine, Siriraj Hospital, Mahidol University, Bangkok 10700 Thailand. ${ }^{12}$ Center for Vaccine Development, Institute of Science and Technology for Research and Development, Mahidol University, 25/25 Moo 3, Phuttamonthon 4 Road, Salaya, Phuttamonthon District, Nakhon Pathom 73170, Thailand. ${ }^{13}$ Génétique, Papillomavirus et Cancer Humain, Institut Pasteur, 25 rue du docteur Roux, 75724 Paris Cedex 15, France. Correspondence should be addressed to C.J. (cjulier@pasteur.fr).
} 
symptomatic individuals with dengue fever (classical incapacitating dengue fever $)^{3}$, excluding those whose only clinical manifestation was fever. Effectively, less severe cases may overlap with undifferentiated fever, which do not require hospitalization and represent, together with asymptomatic cases, most cases of dengue virus infection ( $\sim 90 \%)$ in endemic areas ${ }^{4}$. In this hospital-based recruitment, the frequency of dengue hemorrhagic fever was $74.9 \%$ overall, similar in each hospital; the sex distribution was unbiased; and there was no significant effect of age on disease severity (Supplementary Table 1 online). There was an increased risk of dengue hemorrhagic fever versus dengue fever in individuals who had secondary versus primary dengue infection (odds ratio $(\mathrm{OR})=3.30, P=2 \times 10^{-5}$; Supplementary Table 1 online), as previously reported ${ }^{5}$.

We screened the gene CD209 for polymorphisms, including all exons, part of the introns, 1,091 bp of sequence $5^{\prime}$ to the start codon and 369 bp $3^{\prime}$ to the gene, in 80 Thai individuals (affected individuals and controls). We identified 40 polymorphisms (Supplementary Table 2 online). There was significant linkage disequilibrium (LD) between several of these polymorphisms (Fig. 1), and three haplotypes accounted for $56 \%$ of all haplotypes.

We carried out tests for association with risk of disease (all individuals with dengue disease versus controls) and with severity of disease. Initially, we selected eight polymorphisms for association 을 screening in the first cohort (cohort RA from Ramathibodi Hospital): polymorphisms representative of each LD group (groups defined as pairwise LD index $\Delta>0.75$ ) with rare allele frequency $>0.02$ or that affected the amino acid sequence. We obtained significant results for DCSIGN1-336, which affected disease severity among individuals with dengue classified in three groups (dengue fever, dengue hemorrhagic fever grade I and dengue hemorrhagic fever grades II-IV; $P=0.0022$; data not shown). Because there was no heterogeneity between dengue hemorrhagic fever disease grades (grade I versus grades II-IV; $P=0.53$; data not shown), we considered dengue hemorrhagic fever as a single group in subsequent analyses.

Genotypes GG and GA were rare in individuals with dengue fever (frequency $=2.0 \%$ ) compared with controls (frequency $=18.9 \%$;
$P=0.0012$ ) and were not associated with risk of disease overall (all individuals with dengue disease; Table 1). Genotypes GG and GA were strongly associated with risk of dengue hemorrhagic fever versus dengue fever $\left(\mathrm{OR}=14.31 ; P=2.3 \times 10^{-4}\right)$.

To replicate these results and explore this genetic effect further, we studied these eight polymorphisms and three additional ones in LD with DCSIGN1-336, in this cohort (cohort RA) and two additional independent cohorts (cohorts SI and KK from Siriraj Hospital and Khon-Kaen Hospital, respectively). The results obtained for DCSIGN1-336 in cohort RA were replicated in cohorts SI and KK (Table 2): genotypes GG and GA had a lower frequency in individuals with dengue fever (frequency $=8.3 \%$ and $0.0 \%, P=0.018$ and 0.012 , in cohorts SI and KK, respectively) and were associated with increased risk of dengue hemorrhagic fever versus dengue fever $(P=0.0024$ and 0.037 in cohorts SI and KK, respectively; Table 1). There was no heterogeneity among affected individuals and among controls in the three cohorts or in the odds ratios observed between cohorts $(P=0.42)$. Therefore, the association between CD209 and clinical manifestation of dengue infection is replicated in the three independent cohorts from Thailand.

Overall, we obtained significant association results for DCSIGN1336, located in the CD209 promoter region, and three intronic polymorphisms in LD with it: DCSIGN1.in2+11, DCSIGN1.in5-178 and DCSIGN1.in6-37 ( $\Delta=0.92,0.83$ and 0.53 , respectively), with the most significant results obtained for DCSIGN1-336 (Table 2). For DCSIGN1-336, genotypes GG and GA had a frequency of $4.7 \%$ in individuals with dengue fever and $22.4 \%$ in individuals with dengue hemorrhagic fever. The difference between dengue fever and dengue hemorrhagic fever was highly significant overall $(\mathrm{OR}=5.84$; $\left.P=1.4 \times 10^{-7}\right)$. The frequency of genotypes GG and GA in the control population $(19.5 \%)$ was similar to that in individuals with dengue hemorrhagic fever $(22.4 \% ; P=0.27)$ and different from that in individuals with dengue fever $\left(4.7 \% ; P=2 \times 10^{-6}\right)$. The $P$ values remained highly significant after conservative correction for multiple testing. These genotype differences were similar for individuals with primary and secondary infections (Table 3 ).

Table 1 Association of DCSIGN1-336 in three independent cohorts from Thailand

Genotype distribution

\begin{tabular}{|c|c|c|c|c|c|c|c|c|c|c|}
\hline \multirow[b]{2}{*}{ Cohort and group } & \multicolumn{3}{|c|}{ Number } & \multicolumn{3}{|c|}{ Frequency (\%) } & \multicolumn{2}{|c|}{ Cases versus controls ${ }^{a}$} & \multicolumn{2}{|c|}{ DHF versus $\mathrm{DF}^{\mathrm{a}}$} \\
\hline & $\mathrm{GG}$ & GA & $A A$ & $\mathrm{GG}$ & $\mathrm{GA}$ & AA & OR & $P^{b}$ & OR (95\% c.i.) & $P^{b}$ \\
\hline \multicolumn{11}{|l|}{ RA cohort } \\
\hline Control & 5 & 51 & 240 & 1.7 & 17.2 & 81.1 & & & & \\
\hline DEN & 4 & 39 & 190 & 1.5 & 17.3 & 81.2 & 0.970 & 0.98 & & \\
\hline DF & 0 & 1 & 50 & 0.0 & 2.0 & 98.0 & 0.090 & 0.0012 & $14.31(3.34-61.23)$ & $2.3 \times 10^{-4}$ \\
\hline $\mathrm{DHF}$ & 4 & 38 & 140 & 2.2 & 20.9 & 76.9 & 1.286 & 0.33 & & \\
\hline \multicolumn{11}{|l|}{ SI cohort } \\
\hline Control & 1 & 44 & 169 & 0.5 & 20.6 & 79.0 & & & & \\
\hline DEN & 0 & 49 & 190 & 0.0 & 20.5 & 79.5 & 0.968 & 0.98 & & \\
\hline DF & 0 & 6 & 66 & 0.0 & 8.3 & 91.7 & 0.344 & 0.018 & 3.79 (1.62-8.87) & 0.0024 \\
\hline DHF & 0 & 43 & 124 & 0.0 & 25.7 & 74.3 & 1.302 & 0.34 & & \\
\hline \multicolumn{11}{|l|}{ KK cohort } \\
\hline Control & 3 & 31 & 149 & 1.6 & 16.9 & 81.4 & & & & \\
\hline DEN & 0 & 16 & 114 & 0.0 & 12.3 & 87.7 & 0.616 & 0.18 & & \\
\hline DF & 0 & 0 & 27 & 0.0 & 0.0 & 100.0 & 0.008 & 0.012 & 99.75 (12.70-783.54) & 0.037 \\
\hline DHF & 0 & 16 & 87 & 0.0 & 15.5 & 84.5 & 0.807 & 0.63 & & \\
\hline
\end{tabular}

The SI and KK cohorts were replication cohorts. c.i., confidence interval; DEN, all individuals with dengue disease; DF, dengue fever; DHF, dengue hemorrhagic fever.

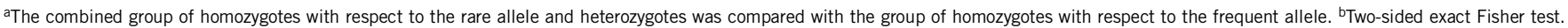


Frequency estimates of haplotypes constructed from the five SNPs in significant LD with DCSIGN1-336 showed that all three haplotypes with a G at DCSIGN1-336 had a lower frequency in individuals with dengue fever than in controls or individuals with dengue hemorrhagic fever (Supplementary Table 3 online). After accounting for DCSIGN1336 , the other polymorphisms were not significantly correlated with disease status. In contrast, the association with DCSIGN1-336 was always significant, even after adjusting for other SNPs in a logistic regression (Table 4 ).

We also investigated whether the observed association could be due to LD between CD209 SNPs and a functional variant located in one of the flanking genes. The two closest flanking genes are CLEC4M (also called L-SIGN or CD209L, liver/lymph node-specific ICAM3-grabbing nonintegrin), located $15.7 \mathrm{~kb}$ proximal to CD209, and CLEC4G (also

\begin{tabular}{|c|c|c|c|c|c|c|c|c|c|c|c|c|}
\hline \multirow{2}{*}{\multicolumn{2}{|c|}{ Polymorphism }} & \multirow[b]{3}{*}{ Group } & \multicolumn{6}{|c|}{ Genotype distribution } & \multirow{2}{*}{\multicolumn{2}{|c|}{ Cases versus controls ${ }^{\mathrm{a}}$}} & \multirow{2}{*}{\multicolumn{2}{|c|}{ DHF versus $\mathrm{DF}^{\mathrm{a}}$}} \\
\hline & & & \multicolumn{3}{|c|}{ Number } & \multicolumn{3}{|c|}{ Frequency (\%) } & & & & \\
\hline Code & Name & & 11 & 12 & 22 & 11 & 12 & 22 & OR & $p^{b}$ & OR (95\% c.i.) & $P^{b}$ \\
\hline \multirow[t]{4}{*}{4} & DCSIGN1-336 & Control & 9 & 126 & 558 & 1.3 & 18.2 & 80.5 & & & & \\
\hline & & DEN & 4 & 104 & 494 & 0.7 & 17.3 & 82.3 & 0.904 & 0.52 & & \\
\hline & & DF & 0 & 7 & 143 & 0.0 & 4.7 & 95.3 & 0.204 & $2.0 \times 10^{-6}$ & $5.84(2.77-12.31)$ & $1.4 \times 10^{-7}$ \\
\hline & & DHF & 4 & 97 & 351 & 0.9 & 21.5 & 77.3 & 1.189 & 0.27 & & \\
\hline \multirow[t]{4}{*}{5} & DCSIGN1-139 & Control & 62 & 310 & 322 & 8.9 & 44.7 & 46.4 & & & & \\
\hline & & DEN & 55 & 244 & 306 & 9.1 & 40.3 & 50.6 & 0.846 & 0.15 & & \\
\hline & & DF & 12 & 57 & 83 & 7.9 & 37.5 & 54.6 & 0.720 & 0.08 & $1.24(0.86-1.79)$ & 1.0 \\
\hline & & DHF & 43 & 187 & 223 & 9.5 & 41.3 & 49.2 & 0.893 & 0.38 & & \\
\hline \multirow[t]{4}{*}{7} & DCSIGN1.in2+11 & Control & 7 & 101 & 585 & 1.0 & 14.6 & 84.4 & & & & \\
\hline & & DEN & 1 & 77 & 524 & 0.2 & 12.8 & 87.0 & 0.806 & 0.20 & & \\
\hline & & DF & 0 & 6 & 146 & 0.0 & 3.9 & 96.1 & 0.224 & $4.5 \times 10^{-5}$ & $4.60(2.07-10.22)$ & $5.4 \times 10^{-5}$ \\
\hline & & DHF & 1 & 71 & 378 & 0.2 & 15.8 & 84.0 & 1.032 & 0.91 & & \\
\hline \multirow[t]{4}{*}{12} & DCSIGN1.ex4SF & Control & 688 & 4 & 0 & 99.4 & 0.6 & 0.0 & & & & \\
\hline & & DEN & 594 & 9 & 0 & 98.5 & 1.5 & 0.0 & 2.588 & 0.17 & & \\
\hline & & DF & 151 & 1 & 0 & 99.3 & 0.7 & 0.0 & 1.181 & 1.0 & $2.61(0.56-12.23)$ & 0.59 \\
\hline & & DHF & 443 & 8 & 0 & 98.2 & 1.8 & 0.0 & 3.087 & 0.10 & & \\
\hline \multirow[t]{4}{*}{13} & DCSIGN1.ex4RPT & Control & 0 & 8 & 685 & 0.0 & 1.2 & 98.8 & & & & \\
\hline & & DEN & 1 & 7 & 595 & 0.2 & 1.2 & 98.7 & 1.151 & 0.97 & & \\
\hline & & DF & 1 & 3 & 148 & 0.7 & 2.0 & 97.4 & 2.328 & 0.31 & $0.33(0.09-1.16)$ & 0.23 \\
\hline & & DHF & 0 & 4 & 447 & 0.0 & 0.9 & 99.1 & 0.771 & 0.91 & & \\
\hline \multirow[t]{4}{*}{15} & DCSIGN1.in5-178 & Control & 6 & 96 & 586 & 0.9 & 14.0 & 85.2 & & & & \\
\hline & & DEN & 1 & 74 & 521 & 0.2 & 12.4 & 87.4 & 0.827 & 0.28 & & \\
\hline & & $\mathrm{DF}$ & 0 & 5 & 144 & 0.0 & 3.4 & 96.6 & 0.201 & $4.0 \times 10^{-5}$ & $5.30(2.25-12.46)$ & $3.0 \times 10^{-5}$ \\
\hline & & DHF & 1 & 69 & 377 & 0.2 & 15.4 & 84.3 & 1.067 & 0.76 & & \\
\hline \multirow[t]{4}{*}{16} & DCSIGN1.ex6TI & Control & 0 & 7 & 679 & 0.0 & 1.0 & 99.0 & & & & \\
\hline & & DEN & 0 & 6 & 589 & 0.0 & 1.0 & 99.0 & 0.989 & 1.0 & & \\
\hline & & DF & 0 & 1 & 148 & 0.0 & 0.7 & 99.3 & 0.683 & 1.0 & $1.61(0.32-8.08)$ & 1.0 \\
\hline & & DHF & 0 & 5 & 441 & 0.0 & 1.1 & 98.9 & 1.103 & 1.0 & & \\
\hline \multirow[t]{4}{*}{17} & DCSIGN1.in6-37 & Control & 6 & 74 & 598 & 0.9 & 10.9 & 88.2 & & & & \\
\hline & & DEN & 1 & 57 & 544 & 0.2 & 9.5 & 90.4 & 0.797 & 0.25 & & \\
\hline & & DF & 0 & 7 & 142 & 0.0 & 4.7 & 95.3 & 0.371 & 0.01 & $2.56(1.19-5.52)$ & 0.02 \\
\hline & & DHF & 1 & 50 & 402 & 0.2 & 11.0 & 88.7 & 0.949 & 0.86 & & \\
\hline \multirow[t]{4}{*}{26} & DCSIGN1.2281 & Control & 287 & 330 & 72 & 41.7 & 47.9 & 10.4 & & & & \\
\hline & & DEN & 227 & 283 & 94 & 37.6 & 46.9 & 15.6 & 1.186 & 0.15 & & \\
\hline & & DF & 49 & 78 & 25 & 32.2 & 51.3 & 16.4 & 1.500 & 0.04 & $0.73(0.50-1.08)$ & 0.14 \\
\hline & & DHF & 178 & 205 & 69 & 39.4 & 45.4 & 15.3 & 1.099 & 0.48 & & \\
\hline \multirow[t]{4}{*}{36} & DCSIGN1.3197 & Control & 11 & 138 & 510 & 1.7 & 20.9 & 77.4 & & & & \\
\hline & & DEN & 8 & 113 & 459 & 1.4 & 19.5 & 79.1 & 0.902 & 0.50 & & \\
\hline & & DF & 3 & 21 & 125 & 2.0 & 14.1 & 83.9 & 0.658 & 0.10 & $1.51(0.93-2.45)$ & 0.12 \\
\hline & & DHF & 5 & 92 & 334 & 1.2 & 21.3 & 77.5 & 0.994 & 1.0 & & \\
\hline \multirow[t]{4}{*}{37} & DCSIGN1.3852 & Control & 353 & 258 & 47 & 53.6 & 39.2 & 7.1 & & & & \\
\hline & & DEN & 285 & 173 & 33 & 58.0 & 35.2 & 6.7 & 0.837 & 0.15 & & \\
\hline & & DF & 71 & 51 & 5 & 55.9 & 40.2 & 3.9 & 0.913 & 0.64 & $0.89(0.59-1.33)$ & 0.64 \\
\hline & & $\mathrm{DHF}$ & 214 & 122 & 28 & 58.8 & 33.5 & 7.7 & 0.811 & 0.13 & & \\
\hline
\end{tabular}

c.i., confidence interval; DEN, all individuals with dengue disease; DF, dengue fever; DHF, dengue hemorrhagic fever.

aThe combined group of homozygotes with respect to the rare allele and heterozygotes was compared with the group of homozygotes with respect to the frequent allele. ${ }^{b}$ Two-sided exact Fisher test. 
Table 3 DCSIGN1-336 in primary and secondary dengue virus infections

\begin{tabular}{|c|c|c|c|c|c|c|}
\hline & \multicolumn{3}{|c|}{$\begin{array}{l}\text { Primary infection } \\
\text { Genotype }\end{array}$} & \multicolumn{3}{|c|}{$\begin{array}{c}\text { Secondary infection } \\
\text { Genotype }\end{array}$} \\
\hline & \multicolumn{2}{|c|}{ Number } & \multirow{2}{*}{$\frac{\text { Frequency (\%) }}{\mathrm{GG}+\mathrm{GA}}$} & \multicolumn{2}{|c|}{ Number } & \multirow{2}{*}{$\frac{\text { Frequency (\%) }}{G G+G A}$} \\
\hline & $\mathrm{GG}+\mathrm{GA}$ & $A A$ & & $G G+G A$ & $\mathrm{AA}$ & \\
\hline DF & 0 & 33 & 0.0 & 7 & 108 & 6.1 \\
\hline DHF & 9 & 26 & 25.7 & 90 & 318 & 22.1 \\
\hline
\end{tabular}

DF, dengue fever; DHF, dengue hemorrhagic fever.

called LSECtin, liver and lymph node sinusoidal endothelial cell C-type lectin), $7.9 \mathrm{~kb}$ distal to CD209 (ref. 6). We sequenced the exons and promoter regions of these two genes in 80 Thai individuals and found limited LD between CD209 and both genes, with $\Delta$ values $<0.75$, except for one SNP located $3^{\prime}$ to CLEC4M that showed strong association with four SNPs in $C D 209(\Delta>0.95)$ that were not associated with disease (Supplementary Fig. 1 online). All $\Delta$ values of CLEC4M and CLEC4G SNPs with DCSIGN1-336 were $<0.27$ (Supplementary Table 4 online). Therefore, it is unlikely that this effect is related to variants in these two flanking genes. Overall, these results support the idea that DCSIGN1-336 is responsible for the observed association with severity of dengue disease.

DCSIGN1-336 is located in the promoter region of CD209, $212 \mathrm{bp}$ $5^{\prime}$ of the major transcription start site $^{7}$. This site affects multiple predicted transcription factor binding sites for Sp1/GATA1/CACCCand CAC-binding transcription factors, of the type GGGTGGG, with allele $\mathrm{G}$ (underlined) associated with the presence of binding sites, and
Table 4 Genetic mapping of the variant responsible for CD209 association with dengue to DCSIGN1-336

\begin{tabular}{lcc}
\hline Second variant & $\begin{array}{c}\text { Residual effect of } \\
\text { DCSIGN1-336a }\end{array}$ & $\begin{array}{c}\text { Residual effect of } \\
\text { second variant }^{\mathrm{b}}\end{array}$ \\
\hline DCSIGN1-139 & $5 \times 10^{-8}$ & 0.44 \\
DCSIGN1.in2+11 & 0.0006 & 0.67 \\
DCSIGN1.ex4SF & $5 \times 10^{-8}$ & 0.31 \\
DCSIGN1.ex4RPT & $7 \times 10^{-8}$ & 0.20 \\
DCSIGN1.in5-178 & 0.002 & 0.93 \\
DCSIGN1.ex6TI & $2 \times 10^{-8}$ & 0.61 \\
DCSIGN1.in6-37 & $6 \times 10^{-7}$ & 0.54 \\
DCSIGN1.2281 & $9 \times 10^{-8}$ & 0.62 \\
DCSIGN1.3197 & $1 \times 10^{-8}$ & 0.21 \\
DCSIGN1.3852 & $4 \times 10^{-8}$ & 0.11 \\
\hline
\end{tabular}

Statistical analysis was done with a likelihood ratio test using logistic regression. a $P$ value testing for residual effect of DCSIGN1-336 when accounting for the effect of the second variant. ${ }^{b} P$ value testing for residual effect of the second variant when accounting for the effect of DCSIGN1-336.

allele A with its absence. This sequence was conserved in the homologous gene $C D 209 L$, with a $G$ at the corresponding position ${ }^{7}$. We carried out electrophoretic mobility shift assays (EMSAs) by incubating HeLa nuclear extracts with two alternative 22-nucleotide probes differing at the DCSIGN1-336 position (Fig. 2a,b). The G variant showed increased Sp1 binding capacity compared with the A variant, which was effectively competed by the $\mathrm{Sp} 1$ consensus oligonucleotide. This suggests that the DCSIGN1-336 variant affects the binding of Sp1 and possibly other transcription factors that modulate transcriptional activity, supporting the idea that is has a functional role in the transcriptional regulation of CD209. It probably does not affect the a

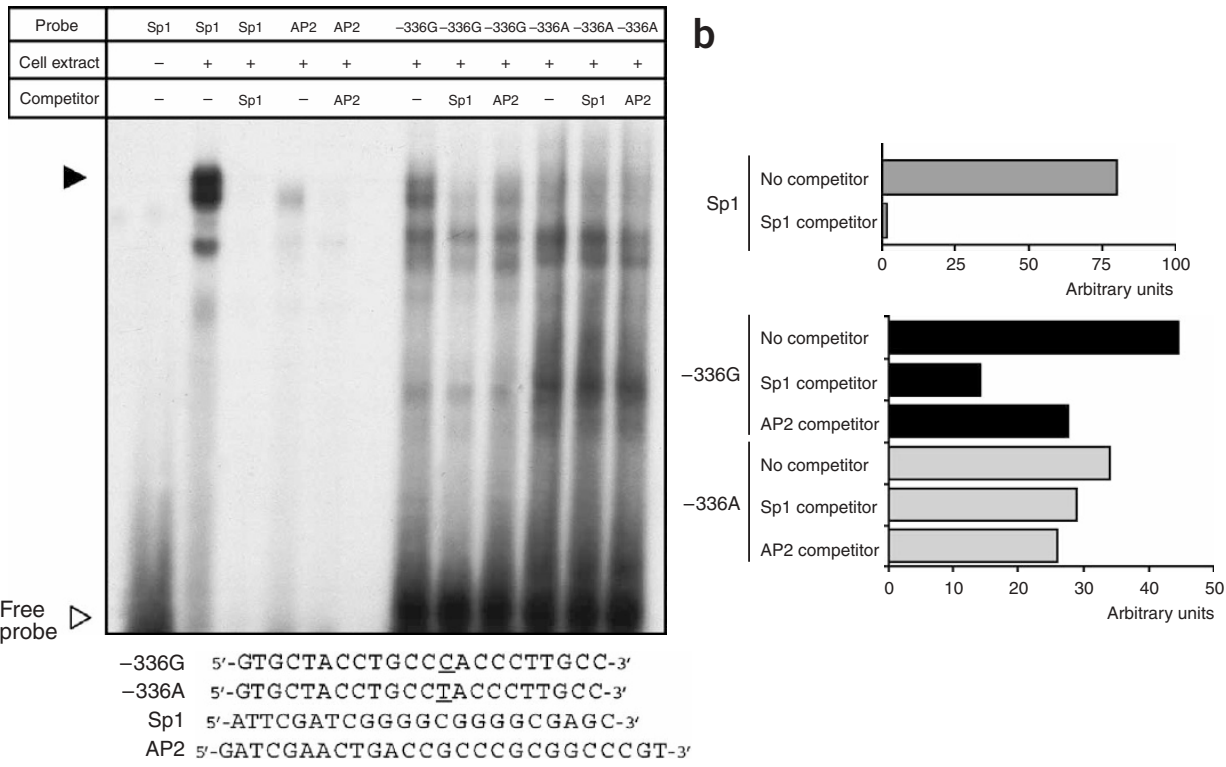

C
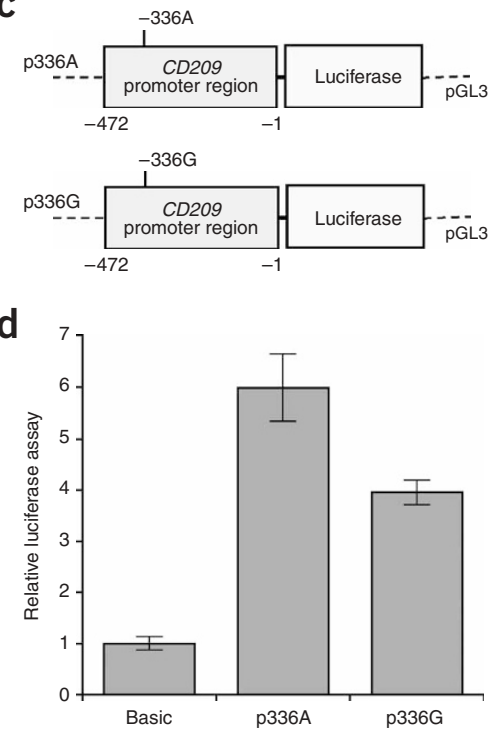

Figure 2 Effects of the DCSIGN1-336 polymorphism on transcriptional activity. (a) EMSAs with ${ }^{32}$ P-labeled probes containing the -336G variant, the $-336 \mathrm{~A}$ variant, the Sp1 consensus site or the AP2 consensus site and HeLa cell nuclear extract. Competition experiments were done in the presence of a tenfold excess of unlabeled Sp1 or AP2 probe. (b) Quantification of the major oligonucleotide-nuclear protein complex (filled arrowhead in panel a). Competition with unlabeled Sp1 consensus probe showed a threefold decrease in binding to the $-336 \mathrm{G}$ probe, but no significant decrease in binding to the 336A probe. The AP2 consensus probe showed slight and similar competition to both probes. (c,d) Luciferase expression of reporter gene constructs expressing sequence from -472 to -1 in transfected HeLa cells. (c) Schematic representation of reporter gene constructs containing the CD209 promoter region with the DCSIGN1-336 polymorphism. (d) Relative luciferase expression from pGL3-Basic, the parental vector without promoter. Luciferase expression from the $C D 209$ reporters relative to this value is shown. 
tissue specificity of expression, which is controlled by sequences outside of the promoter ${ }^{7}$.

We then tested the effect of the DCSIGN1-336 variant on transcription, using two luciferase reporter gene constructs spanning the promoter region from -472 to -1 (CD209 start codon at +1$)$, with either $A$ or $G$ at position -336 , and an invariant nucleotide (G) at position -139 (Fig. 2a,b). Transient transfections of HeLa cells showed that these reporters had promoter function (Fig. 2c,d), confirming previous results ${ }^{7}$. The activity of the $-336 \mathrm{G}$ reporter was significantly lower than that of the $-336 \mathrm{~A}$ reporter $(-336 \mathrm{~A} / \mathrm{G}$ ratio $=1.5, P=10^{-5}$; Fig. $\left.2 \mathbf{c}, \mathbf{d}\right)$, suggesting that the DCSIGN1-336 variant affects promoter activity.

We show that a promoter variant in CD209, DCSIGN1-336, is strongly associated with the risk of developing dengue fever versus dengue hemorrhagic fever and controls. DCSIGN1-336 affects an Sp1 binding site and the level of transcription of CD209 in vitro, which supports the idea that this variant has a functional role. Polymorphisms in Sp1 binding sites have been hypothesized to modify phenotypes or susceptibility to diseases such as bone density and osteoporosis $^{8,9}$, estrogen response $\mathrm{e}^{10}$ and acute myeloid leukemia ${ }^{11}$.

On the basis of these results, we propose that the $G$ allele at DCSIGN1-336 is associated with a dominant protection against dengue fever $\left(\mathrm{OR}=4.90 ; P=2 \times 10^{-6}\right)$ but not against dengue 윽 hemorrhagic fever. Among individuals with dengue, genotypes GG and GA strongly increased the risk of contracting dengue hemorrhagic fever versus dengue fever $\left(\mathrm{OR}=5.84 ; P=1.4 \times 10^{-7}\right)$. Therefore, O there is strong evidence of genetic heterogeneity between individuals with dengue fever and dengue hemorrhagic fever.

Our genetic results suggest that dengue fever and dengue hemorrhagic fever, two severe forms of dengue disease, may involve pathophysiological processes that are at least partially distinct, with different levels of involvement of CD209 and possibly other host and viral factors. These findings bring new insight to the classical view of a continuum of disease severity between dengue fever and dengue hemorrhagic fever ${ }^{12}$. The expected decrease in expression of CD209 (2) carrying the G allele at DCSIGN1-336 may result in a lower susceptibility of dendritic cells to dengue virus in the early stages of infection $^{1,2}$, which would protect against dengue fever, whereas other pathophysiological processes would be prevalent in dengue hemorrhagic fever. It is notable that major disturbances of immune responses are observed in dengue hemorrhagic fever compared with dengue fever ${ }^{13}$.

We propose two possible hypotheses to explain the selective protection of the CD209 variant against dengue fever but not dengue hemorrhagic fever. First, there may be differential interaction between dengue virus and CD209, depending on the genetic variability of the dengue virus strain, which affects both the level of infectivity of CD209-expressing dendritic cells ${ }^{2}$ and the viral virulence ${ }^{14}$. Second, in dengue hemorrhagic fever, there may be an alternative pathway to the CD209-mediated dengue virus entry to dendritic cells. The high viral load observed in dengue hemorrhagic fever may be caused by the antibody-enhanced dengue virus infection of monocytes through $\mathrm{F} c \gamma$ receptors in individuals with pre-existing dengue virus antibodies (antibody-dependant enhancement). Previous studies have reported some association of polymorphisms in HLA class I, TNF $\alpha$ and Fc $\gamma$ receptor IIA genes with dengue hemorrhagic fever ${ }^{15-20}$. Notably, some HLA alleles were specifically associated with the risk of dengue fever rather than dengue hemorrhagic fever ${ }^{18}$. Overall, the risk of infection and disease severity probably results from complex interactions between CD209 variability and other host and viral factors, whose elucidation requires further detailed investigations.
Our conclusion that the CD209 variant DCSIGN1-336 has a role in disease orientation (dengue fever or dengue hemorrhagic fever) may have consequences for the development of CD209-based prophylaxis and therapy against infections by dengue virus and a wide variety of pathogens of medical relevance. In addition to its role in infection by arboviruses such as dengue virus ${ }^{21,22}$, CD209 is an attachment factor for many other pathogens: viruses with glycosylated envelope proteins, including human immunodeficiency virus-1, hepatitis $\mathrm{C}$ virus, cytomegalovirus, Ebola virus and SARS-coV; bacteria such as Mycobacterium tuberculosis; and parasites such as Leishmania and Schistosoma mansoni ${ }^{22-25}$. DCSIGN1-336 (and possibly other genetic variants of CD209) may therefore have a role in the susceptibility to other infectious diseases, as suggested for susceptibility to parenteral HIV-1 infection ${ }^{26}$. Host response to medical vaccines and drugs in terms of efficiency and safety might depend on genotype with respect to CD209 polymorphisms, which should be taken into account to optimize the therapeutic designs.

\section{METHODS}

Patients and controls. We recruited individuals with dengue disease and controls independently at three medical centers in Thailand: two in Bangkok, Ramathibodi Hospital (cohort RA) and Siriraj Hospital (cohort SI), Mahidol University, and one in the northeast region of Thailand, Khon-Kaen Hospital (cohort KK). Diagnosis was established by expert clinicians in each center, following World Health Organization criteria as follows. Individuals suspected to have dengue viral infection on the basis of clinical features including high fever, severe headache, retro-orbital pain, myalgia, arthalgia, nausea and vomiting, and rash were admitted to the hospitals for clinical observation and treatment. The diagnosis of dengue virus infection was later confirmed by a comparable dengue-specific IgG and IgM enzyme-linked immunosorbent assay titer on a late acute or convalescent sera ${ }^{27}$. Differential diagnosis of dengue fever and dengue hemorrhagic fever was established on the basis of the absence (dengue fever) or presence (dengue hemorrhagic fever) of evidence for increased vascular permeability manifested by hemoconcentration or pleural effusion. Specifically, the diagnosis of dengue hemorrhagic fever was made on the basis of the four following characteristics: (i) high continuous fever lasting 2-7 d, (ii) hemorrhagic tendency such as a positive tourniquet test, petechii, purpura or hematemesis, (iii) thrombocytopenia (platelet count $\geq 100,000 \mu \mathrm{l}^{-1}$ and (iv) evidence of plasma leakage due to increased vascular permeability manifested by hemoconcentration (an increased in hematocrit of $20 \%$ or more) or pleural effusion. The severity of dengue hemorrhagic fever was categorized in four grades according to World Health Organization criteria ${ }^{3}$. Grades III and IV were dengue hemorrhagic fever with narrowing pulse pressure with a characteristically elevated diastolic pressure to profound shock. In the three cohorts, clinical and biological data were recorded during their hospitalization and stored into a database.

In addition to the common criteria used for diagnosis, there were specific features in each center. At Ramathibodi Hospital, but not in other centers, a chest X-Ray in right lateral decubitus was done in individuals without evidence of hemoconcentration to refine the differential diagnosis of dengue hemorrhagic fever versus dengue fever on the basis of pleural effusion. At Ramathibodi Hospital, affected individuals were requested to come back to the hospital 2-3 weeks after discharge, and a follow-up hematocrit and platelet measure was done, allowing for better evaluation of increase in hematocrit during previous admission. At Khon-Kaen Hospital, clinical and biological recording was more systematic and was computerized from the start of the study, and this detailed information was used to complement the dengue status established by clinicians. In cohort KK, hematocrit measurements were done at least four times a day, and up to every hour during the critical period of defeverescence. Because of the detailed biological data collected in this cohort, we made the differential diagnosis of dengue hemorrhagic fever grade I versus dengue fever on the basis of evidence of plasma leakage (increased hematocrit of $20 \%$ or more), whereas dengue hemorrhagic fever grades II-IV was established by the clinicians. Secondary infection was defined as a dengue-specific IgM/IgG ratio $<1.8$. 
To preserve homogeneity, we included only school-age children (between the ages of 5 and 15 years, inclusive). To limit heterogeneity among individuals with dengue fever, we excluded from our study those presenting with fever as the only clinical manifestation. In practice, only children with dengue fever manifesting at least one of the following diagnosis criteria, in addition to fever, were included in the dengue fever group: rash, bleeding manifestations (including petechiae) or hepatomegaly. These most closely reflect classical incapacitating dengue fever ${ }^{3}$.

We studied a total of 606 affected individuals: 235,241 and 130 in cohorts RA, SI and KK, respectively; 301 males and 305 females.

Controls consisted of a total of 696 healthy blood donors, 296, 216 and 184 recruited from Ramathibodi Hospital, Siriraj Hospital and Khon-Kaen Hospital, respectively ( $50 \%$ female). For Ramathibodi Hospital and Siriraj Hospital, both cases and control groups came from Bangkok and the central part of Thailand; for Khon-Kaen Hospital, both came from the northeast region of Thailand. We collected whole blood samples on EDTA from affected individuals and controls and extracted DNA using a standard phenolchloroform method.

We obtained signed informed consent from all participants or their tutors, and ethical approvals were granted by the ethical committees from the Faculty of Medicine, Ramathibodi Hospital, Mahidol University; the Faculty of Medicine, Siriraj Hospital, Mahidol University; the Khon Kaen Hospital and the Ministry of Public Health.

Polymorphism identification and genotyping. We identified polymorphisms in CD209 by direct sequencing of PCR-amplified genomic DNA from 80 Thai individuals: 48 individuals with dengue ( 25 with dengue fever and 23 with dengue hemorrhagic fever) and 32 controls. Primers used for sequencing CD209 exons, part of introns, and $5^{\prime}$ and $3^{\prime}$ regions are listed in Supplementary Table 5 online. Similarly, we identified polymorphisms in CLEC4M and CLEC4G in 48 individuals with dengue and 32 controls using primers shown in Supplementary Table 5 online. We analyzed sequencing results by Genalys Software ${ }^{28}$. We also carried out a direct investigation of a repeated region in exon 4 (ref. 29) in 96 cases and 96 controls, which extended the identification of DCSIGN1.ex4RPT and DCSIGN1.ex4SF polymorphisms. Only three rare variants $(<2 \%)$ affected the coding regions of the protein: two amino acid substitutions and one variation in the number of repeats of a 23 -amino-acid unit located in the predicted extracellular domain ${ }^{29}$ (Supplementary Table 2 online). The other polymorphisms were located in introns (8), in the promoter or $5^{\prime}$ region of the gene (6), in the $3^{\prime}$ untranslated region (21) or $3^{\prime}$ to the gene (2). Sixteen of these polymorphisms were located in repeat sequences. Twenty20 seven of these polymorphisms had a rare allele frequency $>0.02$. We genotyped DCSIGN1-336, DCSIGN1-139, DCSIGN1.in2+11, DCSIGN1.ex4SF and DCSIGN1.2281 using PCR-RFLP assays and DCSIGN1.ex4RPT by PCR and agarose gel electrophoresis (Supplementary Table 6 online). We genotyped DCSIGN1-336, DCSIGN1.in5-178, DCSIGN1.ex6TI, DCSIGN1.in6-37, DCSIGN1.3197 and DCSIGN1.3852 by TaqMan assay (Supplementary Table 7 online) using ABI Prism 7000 Sequence Detection System, with recommended protocols. We genotyped DCSIGN1-336 in duplicate, using both methods, with identical results. We tested deviation from Hardy-Weinberg equilibrium in individuals with dengue disease and in controls, in each cohort and overall, and found that it was not statistically significant for any SNP. We carried out heterogeneity testing for the origin of cohort for each polymorphism in individuals with dengue disease and in controls and found that it was not significant.

Statistical analyses. We carried out tests for association and LD using $\chi^{2}$ or two-sided Fisher exact tests. We estimated haplotype frequencies using the EM algorithm, as implemented in the HAPLO program (S. Heath, unpublished data). We calculated the LD index $(\Delta)$ using all polymorphisms identified in up to 80 Thai individuals with dengue disease and controls ( 25 with dengue fever, 23 with dengue hemorrhagic fever and 32 controls), using the ldmax program, as implemented in the GOLD package ${ }^{30}$. We carried out fine mapping of the responsible variant using logistic regression, comparing likelihoods of onevariant models to two-variants models, using STATVIEW statistic package.

Transcription factor binding sites prediction. We used the TESS interface to predict and compare binding sites in alternative alleles.
EMSA. We detected the DNA-protein complexes by EMSA using the Gel Shift Assay System kit (Promega). We end-labeled the double-stranded DNA probes corresponding to the A and G alleles of variant DCSIGN1-336 (-336A probe and $-336 \mathrm{G}$ probe, respectively) with $\left(\gamma_{-}{ }^{32} \mathrm{P}\right)$ ATP, incubated them with HeLa nuclear extract (Promega) and separated them on a $4 \%$ nondenaturing acrylamide gel. We established the specificity of the DNA binding site by preincubating the HeLa nuclear extract with an excess of unlabeled competitor probes corresponding to the consensus $\mathrm{Sp} 1$ and AP2 sites. All DNA probes sequences are shown in Figure 2a. We analyzed band intensity with PhosphoImager (Molecular Dynamics).

Reporter plasmid construction, plasmid transfection and reporter gene assays. To construct CD209 promoter reporters, we amplified fragments encompassing nucleotides -472 to -1 (relative to the ATG start codon at +1 ) by PCR from genomic DNA of two individuals homozygous with respect to the corresponding genotypes, using primers tailed with BglII and HindIII restriction sites, and subcloned them into the BglII and HindIII sites of the pGL3Basic expression vector (Promega). We verified all recombinant clones by DNA sequencing. We cultured HeLa cells on 12-well culture plates $\left(2 \times 10^{5}\right.$ cells per well). After $1 \mathrm{~d}$ of culture, cell monolayers were transfected for $40 \mathrm{~h}$ with $4 \mu \mathrm{g}$ of plasmid per well in the presence of FuGene 6 transfection reagent (Roche Molecular Biochemicals). After the transfection period, we lysed cells with $0.5 \mathrm{ml}$ of reporter lysis buffer (Promega), incubated them on ice for $10 \mathrm{~min}$ and subjected the lysates to centrifugation at 6,000 r.p.m. for $3 \mathrm{~min}$. We assayed $10 \mu \mathrm{l}$ of supernatant for luciferase activity using the Promega luciferase assay system. We carried out luciferase assays in quadruplicate in at least three independent transfection experiments.

URL. The TESS interface is available at http://www.cbil.upenn.edu/tess/.

Note: Supplementary information is available on the Nature Genetics website.

\section{ACKNOWLEDGMENTS}

We thank A. Chunharas, P. Kitpoka and A. Chairunsri for help in recruiting affected individuals and controls and L. Damrikarnlerd and S. Swasdiworn for managing the clinical database. This work was supported by the Senior Research Scholarship Program of the Thailand Research Fund (P.M.), the Thailand Tropical Disease Research Program T2 (P.M.), the Thailand National Center for Genetic Engineering and Biotechnology (P.M., P.Y. and N.T.), Mahidol University (A.S.), the Thailand SNP Discovery Program (T.L.), the Direction Générale pour l'Armement (P.D.), the Medical Scholar Program, Mahidol University and the Split Mode PhD program (S.M.K.), and the Royal Golden Jubilee Program, the Thailand Research Fund and the French Embassy of Thailand (C.T.).

\section{COMPETING INTERESTS STATEMENT}

The authors declare that they have no competing financial interests.

Received 23 December 2004; accepted 16 March 2005 Published online at http://www.nature.com/naturegenetics/

1. Tassaneetrithep, B. et al. DC-SIGN (CD209) mediates dengue virus infection of human dendritic cells. J. Exp. Med. 197, 823-829 (2003).

2. Navarro-Sanchez, E. et al. Dendritic-cell-specific ICAM3-grabbing non-integrin is essential for the productive infection of human dendritic cells by mosquito-cell-derived dengue viruses. EMBO Rep. 4, 1-6 (2003).

3. World Health Organization. Dengue Haemorrhagic Fever: Diagnosis, Treatment, Prevention and Control (World Health Organization, Geneva, 1997).

4. Burke, D.S., Nisalak, A., Johnson, D.E. \& Scott, R.M. A prospective study of dengue infections in Bangkok. Am. J. Trop. Med. Hyg. 38, 172-180 (1988).

5. Halstead, S.B. Pathogenesis of dengue: challenges to molecular biology. Science 239, 476-481 (1988).

6. Liu, W. et al. Characterization of a novel C-type lectin-like gene, LSECtin: demonstration of carbohydrate binding and expression in sinusoidal endothelial cells of liver and lymph node. J. Biol. Chem. 279, 18748-18758 (2004).

7. Liu, H., Yu, W., Liou, L.Y. \& Rice, A.P. Isolation and characterization of the human DC-SIGN and DC-SIGNR promoters. Gene 313, 149-159 (2003).

8. Grant, S.F. et al. Reduced bone density and osteoporosis associated with a polymorphic Sp1 binding site in the collagen type I alpha 1 gene. Nat. Genet. 14, 203205 (1996).

9. Mann, V. et al. A COL1A1 Sp1 binding site polymorphism predisposes to osteoporotic fracture by affecting bone density and quality. J. Clin. Invest. 107, 899-907 (2001). 
LETTERS

10. Harendza, S. et al. Linked common polymorphisms in the gelatinate a promoter are associated with diminished transcriptional response to estrogen and genetic fitness. J. Biol. Chem. 278, 20490-20499 (2003).

11. Sibley, K. et al. Functional FAS promoter polymorphisms are associated with increased risk of acute myeloid leukemia. Cancer Res. 63, 4327-4330 (2003).

12. Rothman, A.L. Immunology and immunopathogenesis of dengue disease. Adv. Virus Res. 60, 397-419 (2003).

13. Mongkolsapaya, J. et al. Original antigenic sin and apoptosis in the pathogenesis of dengue hemorrhagic fever. Nat. Med. 9, 921-927 (2003).

14. Vaughn, D.W. et al. Dengue viremia titer, antibody response pattern, and virus serotype correlate with disease severity. J. Infect. Dis. 181, 2-9 (2000).

15. Lake, H. et al. Strong HLA class I-restricted T cell responses in dengue hemorrhagic fever: a double-edged sword? J. Infect. Dis. 184, 1369-1373 (2001).

을 6. Chiewsilp, P., Scott, R.M. \& Bhamarapravati, N. Histocompatibility antigens and dengue hemorrhagic fever. Am. J. Trap. Med. Hyg. 30, 1100-1105 (1981).

17. Paradoa Perez, M.L., Trujillo, Y. \& Basanta, P. Association of dengue hemorrhagic fever with the HLA system. Haematologia (Budap.) 20, 83-87 (1987).

18. Stephens, H.A. et al. HLA-A and -B allele associations with secondary dengue virus infections correlate with disease severity and the infecting viral cerotype in ethnic Thais. Tissue Antigens 60, 309-318 (2002).

19. Like, H. et al. Susceptibility to dengue hemorrhagic fever in vietnam: evidence of an association with variation in the vitamin d receptor and Ic gamma receptor Ila genes. Am. J. Trap. Med. Hg. 67, 102-106 (2002).

20. Fernandez-Mestre, M.T., Gendzekhadze, K., Rivas-Vetencourt, P. \& Layrisse, Z. TNFalpha-308A allele, a possible severity risk factor of hemorrhagic manifestation in dengue fever patients. Tissue Antigens 64, 469-472 (2004).

21. Klimstra, W.B., Mangle, E.M., Smith, M.S., Yurochko, A.D. \& Ryman, K.D. DC-SIGN and L-SIGN can act as attachment receptors for alphaviruses and distinguish between mosquito cell- and mammalian cell-derived viruses. J. Virol. 77, 12022-12032 (2003).

22. van Kooyk, Y., Appelmelk, B. \& Geijtenbeek, T.B. A fatal attraction: Mycobacterium tuberculosis and HIV -1 target DC-SIGN to escape immune surveillance. Trends Mol. Med. 9, 153-159 (2003)

23. Halary, F. et al. Human cytomegalovirus binding to DC-SIGN is required for dencritic cell infection and target cell trans-infection. Immunity 17, 653-664 (2002).

24. Tailleux, L. et al. DC-SIGN is the major Mycobacterium tuberculosis receptor on human dendritic cells. J. Exp. Med. 197, 121-127 (2003).

25. Yang, Z.Y. et al. pH-dependent entry of severe acute respiratory syndrome coronavirus is mediated by the spike glycoprotein and enhanced by dendritic cell transfer through DC-SIGN. J. Virol. 78, 5642-5650 (2004).

26. Martin, M.P. et al. Association of DC-SIGN promoter polymorphism with increased risk for parenteral, but not mucosal, acquisition of human immunodeficiency virus type 1 infection. J. Viral. 78, 14053-14056 (2004).

27. Anis, B.L. et al. An enzyme-linked immunosorbent assay to characterize dengue infections where dengue and Japanese encephalitis co-circulate. Am. J. Trap. Med. Hyg. 40, 418-427 (1989).

28. Takahashi, M., Matsuda, F., Margetic, N. \& Lathrop, M. Automated identification of single nucleotide polymorphisms from sequencing data. J. Bioinform. Compute. Biol. 1, 253-265 (2003).

29. Mummidi, S. et al. Extensive repertoire of membrane-bound and soluble dendritic cellspecific ICAM-3-grabbing nonintegrin 1 (DC-SIGN1) and DC-SIGN2 isoforms. Interindividual variation in expression of DC-SIGN transcripts. J. Biol. Chem. 276, 33196 33212 (2001).

30. Abecasis, G.R. \& Cookson, W.O. GOLD-graphical overview of linkage disequilibrium. Bioinformatics 16, 182-183 (2000).

(20)

NATURE GENETICS VOLUME 37 | NUMBER 5 | MAY 2005

513 